\title{
Synthesis and reactions of 8-allylchromone-3-carboxaldehyde
}

\author{
Salah S. Ibrahim, Hassan A. Allimony, Ali M. Abdel-Halim, and \\ Magdy A. Ibrahim* \\ Department of Chemistry, Faculty of Education, Ain Shams University, \\ Roxy, 11711, Cairo-Egypt \\ E-mail: magdyahmed1977@yahoo.com
}

\begin{abstract}
8-Allylchromone derivatives 5-7, 10 and 11 were prepared starting from 8-allylchromone-3carboxaldehyde 4. Basic rearrangement of oxime 8 and/or carbonitrile 9 gave 8-allyl-2aminochromone-3-carboxaldehyde 12. Chromeno[2,3-b] pyridines 14-20 were prepared via the reaction of 12 with malononitrile, cyanoacetamide, ethyl cyanoacetate, phenylthioacetonitrile, ethyl acetoacetate, ethyl benzoylacetate and barbituric acid. Structures of the new products have been deduced from elemental analysis and spectral data (IR, ${ }^{1} \mathrm{H}$ NMR and mass spectra).
\end{abstract}

Keywords: Synthesis, chromone, chromeno[2,3-b]pyridine, Friedländer

\section{Introduction}

Chromone derivatives drew much attention because of their activity against the human immunodeficiency virus (HIV-1) $)^{1-3}$ and their broad anti-inflammatory, ${ }^{4}$ antitumor, ${ }^{5}$ antibacterial, ${ }^{6}$ antimicrobial, ${ }^{7}$ antifungal,${ }^{8,9}$ antibiotic, ${ }^{10}$ and insecticidal activities. ${ }^{11}$ Chromones bearing an allyl group at position 8 have a special medicinal importance; 8-allyl-2styrylchromones were used as inhibitors for the growth of tumors. ${ }^{12}$ Also, the 8 -allyl derivatives were used as a precursor for the synthesis of the 8-acetic acid derivatives which exhibit anticancer properties. ${ }^{13-15}$ Heteroannulated chromones showed significant biological activity including pharmacological, ${ }^{16}$ anti-inflammatory and antiplatelet activities. ${ }^{17}$

\section{Results and Discussion}

In the course of the present work, some new chromone derivatives bearing the allyl group at position 8 have been synthesized starting from 8 -allylchromone-3-carboxaldehyde 4 . The synthetic route of compound 4 is depicted in Scheme 1. Standard procedures were used to convert 2-hydroxyacetophenone $\mathbf{1}$ to its allyl ether derivative $\mathbf{2}$ which under Claisen 
rearrangement gave 3-allyl-2-hydroxyacetophenone $3 .{ }^{14}$ Formylation of $\mathbf{3}$ by Vilsmeier reagent $\left(\mathrm{DMF} / \mathrm{POCl}_{3}\right)$ afforded the target compound 4 . The structure of compound $\mathbf{4}$ was confirmed based on correct elemental analysis and spectral data. Its IR spectrum showed two characteristic absorption bands at $1700\left(\mathrm{C}=\mathrm{O}_{\text {aldehyde }}\right)$ and $1647\left(\mathrm{C}=\mathrm{O}_{\gamma \text {-pyrone }}\right) \mathrm{cm}^{-1}$. The ${ }^{1} \mathrm{H}$ NMR spectrum showed characteristic signals of the allyl segment at $\delta 3.62,5.06$ and $6.05 \mathrm{ppm}$, in addition to two singlet at $\delta 8.93$ and $10.12 \mathrm{ppm}$ attributed to $\mathrm{H}-2$ and the aldehydic proton, respectively.

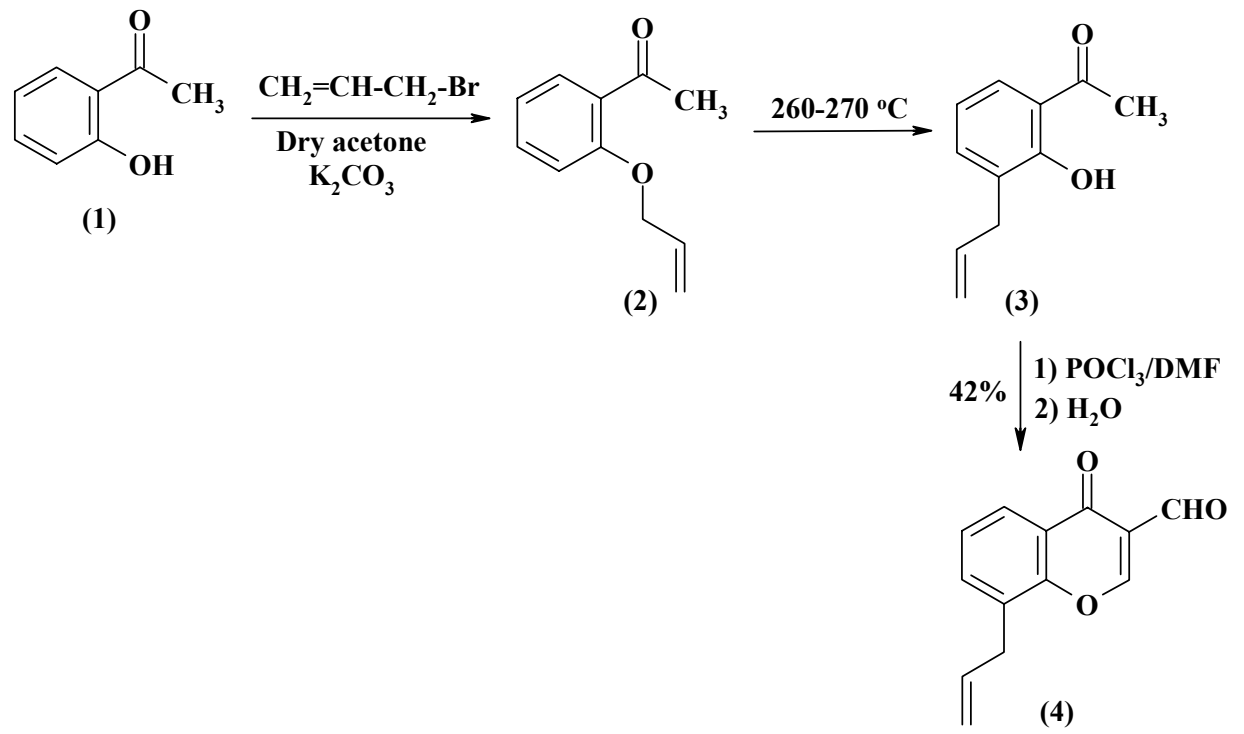

Scheme 1. Synthetic route to 8-allylchromone-3-carboxaldehyde 4.

Condensation of 8-allylchromone-3-carboxaldehyde 4 with hippuric acid in boiling acetic anhydride containing freshly fused sodium acetate gave the oxazolone derivative $\mathbf{5}$ (Scheme 2). ${ }^{18}$ The IR spectrum of 5 showed two characteristic absorption bands at $1803\left(\mathrm{C}=\mathrm{O}_{\text {oxazolone }}\right)$ and $1657\left(\mathrm{C}=\mathrm{O}_{\gamma-\text { pyrone }}\right) \mathrm{cm}^{-1}$. Its ${ }^{1} \mathrm{H}-\mathrm{NMR}$ spectrum exhibited two characteristic singlet signals at $\delta$ 8.21 and $9.75 \mathrm{ppm}$ assigned to the $\mathrm{H}-2$ and olefinic proton, respectively.

Also, condensation of 4 with barbituric acid in dry pyridine gave 5-(8-allylchromon-3ylmethylene)-1,3-dihydropyrimidine-2,4,6-trione 6 (Scheme 2). Its ${ }^{1} \mathrm{H}$ NMR spectrum showed two singlet signals at $\delta 8.44$ and 9.77 assigned to $\mathrm{H}-2$ and the olefinic proton, respectively, in addition to two exchangeable signals at $\delta 11.37$ and $11.47 \mathrm{ppm}$ attributed to $2 \mathrm{NH}$ protons. On the other hand, treatment of carboxaldehyde 4 with dimedone in dry pyridine gave 3,3,6,6tetramethyl-9-(8-allylchromon-3-yl)-1,2,3,4,5,6,7,8-octahydroxanthene-1,8-dione 7 (Scheme 2). When compound 4 was allowed to react with hydroxylamine hydrochloride in boiling ethanol, the oxime 8 was easily obtained. Dehydration of the latter compound by acetic anhydride afforded the corresponding carbonitrile 9 (Scheme 3). 


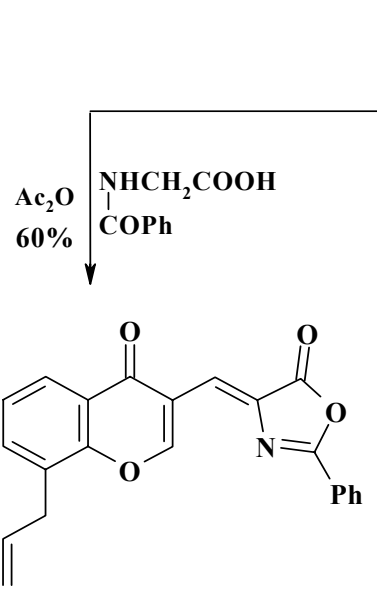

(5)

(4)

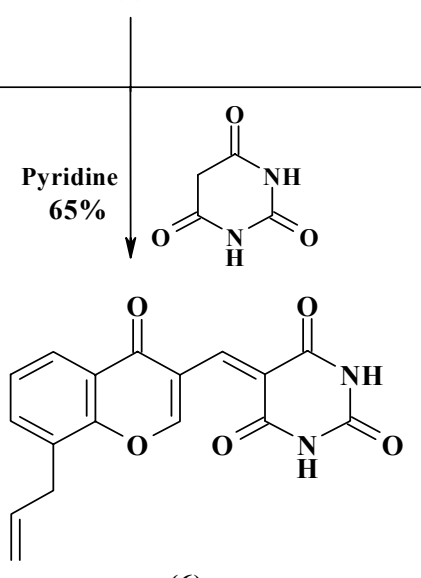

(6)

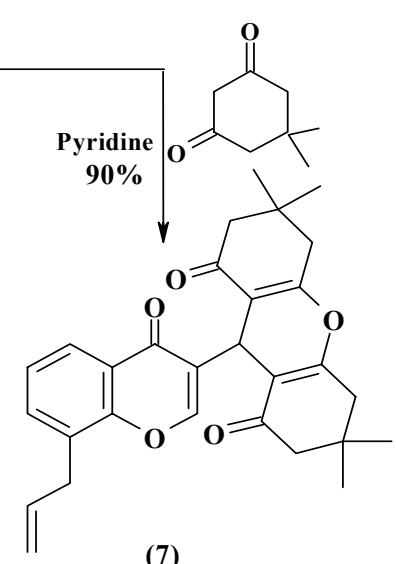

(7)

Scheme 2. Formation of 3-substituted-8-allylchromones 5-7.

It has been reported that chromone derivatives bearing an acidic groups at position 3 displayed antiallergic activities. ${ }^{19,20}$ Thus, in the present work some analogous compounds containing the allyl moiety at position 8 were prepared. Reaction of the carbonitrile 9 with sodium azide in the presence of aluminum chloride in tetrahydrofuran afforded 8-allyl-3- $(1 \mathrm{H}-$ tetrazol-5-yl)chromone 10. ${ }^{21}$ Also, trans 3-(8-allylchromon-3-yl)acrylic acid 11 was prepared via the reaction of 8-allylchromone-3-carboxaldehyde 4 with malonic acid in dry pyridine (Scheme 3). The IR spectrum of compound $\mathbf{1 0}$ exhibited characteristic absorption bands at 3192 $\left(\mathrm{NH}_{\text {tetrazole }}\right)$ and $1644\left(\mathrm{C}=\mathrm{O}_{\gamma-\text { pyrone }}\right) \mathrm{cm}^{-1}$, while compound 11 displayed bands at 3200-2500 $(\mathrm{OH})$, $1701\left(\mathrm{C}=\mathrm{O}_{\text {carboxy }}\right)$ and $1652\left(\mathrm{C}=\mathrm{O}_{\gamma-\text { pyrone }}\right) \mathrm{cm}^{-1}$.<smiles>C=CCc1cccc2c(=O)c(-c3nnn[nH]3)coc12</smiles>

Scheme 3. Formation of 3-substituted-8-allylchromones 8-11. 
On the other hand, the action of $0.05 \mathrm{M}$ sodium hydroxide solution or concentrated ammonium hydroxide solution on 8-allylchromone-3-carboxaldehyde-oxime $\mathbf{8}$ or 8allylchromone-3-carbonitrile 9 results in opening of the $\gamma$-pyrone ring followed by recyclization to give 8-allyl-2-aminochromone-3-carboxaldehyde 12 (Scheme 4). ${ }^{22}$ Using ammonium hydroxide solution gave a superior yield and pure product. The IR spectrum of $\mathbf{1 2}$ showed characteristic absorption bands at 3304, $3175\left(\mathrm{NH}_{2}\right), 1665\left(\mathrm{C}=\mathrm{O}_{\text {aldehyde }}\right)$ and $1635\left(\mathrm{C}=\mathrm{O}_{\gamma \text {-pyrone }}\right)$ $\mathrm{cm}^{-1}$; its ${ }^{1} \mathrm{H}$ NMR spectrum showed signals at $\delta 9.62$ and $10.13 \mathrm{ppm}$ assigned to $\mathrm{NH}_{2}$ and $\mathrm{CHO}$ protons, respectively. The structure was further confirmed from its mass spectrum, which exhibited the molecular ion peak at $\mathrm{m} / \mathrm{e} 229$ and the base peak at $\mathrm{m} / \mathrm{e} 201$. Treatment of $\mathbf{1 2}$ with hydroxylamine hydrochloride in ethanol yielded 8-allyl-2-aminochromone-3-carboxaldehydeoxime 13.

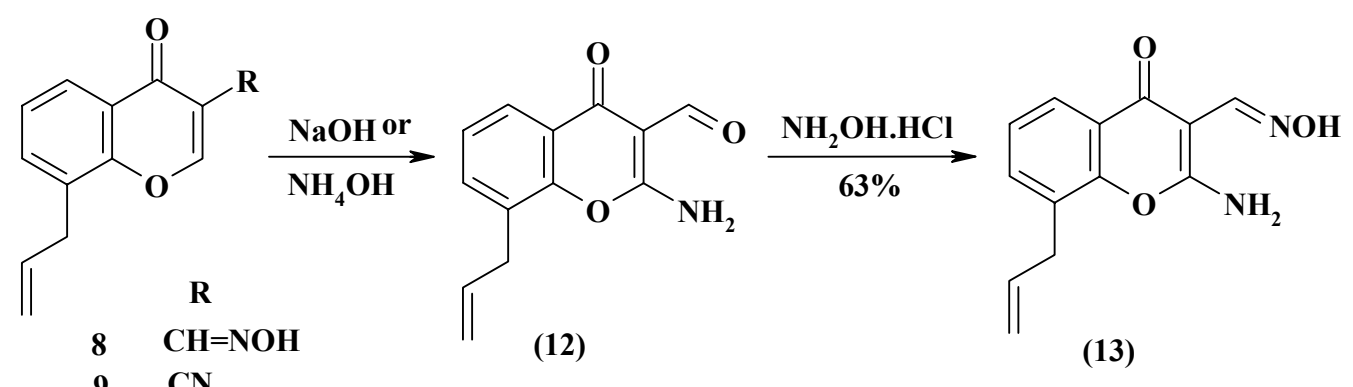

Scheme 4. Synthesis of aminoaldehyde 12 and its corresponding oxime 13.

In continuation to our interest to prepare chromeno[2,3- $b$ ]pyridines, ${ }^{23,24}$ some new chromeno[2,3-b]pyridines 14-20 were prepared successfully from the reaction of 8-allyl-2aminochromone-3-carboxaldehyde $\mathbf{1 2}$ with active methylene compounds in the presence of 1,8diazabicyclo[5.4.0] undec-7-ene (DBU) as a basic catalyst. Reaction of $\mathbf{1 2}$ with active methylene compounds containing a cyano group adjacent to a methylene group $\left(-\mathrm{CH}_{2} \mathrm{CN}\right)$ namely: malononitrile, cyanoacetamide, ethyl cyanoacetate and phenylthioacetonitrile in absolute ethanol containing few drops of DBU afforded 9-allyl-2-amino-5-oxo-5H-chromeno[2,3- $b]$ pyridines 1417, respectively, through condensation followed by cyclo-addition reactions (Scheme 5). Structures of compounds 14-17 were inferred from their correct elemental analysis and spectral data. The IR spectra of compounds 14-17 displayed characteristic absorption bands in the range 3458- 3173 and $1667-1653 \mathrm{~cm}^{-1}$ due to the stretching frequencies of $\mathrm{NH}_{2}$ groups and $\mathrm{C}=\mathrm{O}$ of the $\gamma$-pyrone systems, respectively. The ${ }^{1} \mathrm{H}$ NMR spectra of 14-17 showed characteristic singlet signals in the range $\delta 8.81-8.36 \mathrm{ppm}$ due to $\mathrm{H}-4$ protons, the amino protons were observed in the range $\delta 8.49-7.53 \mathrm{ppm}$. Also, the spectrum of $\mathbf{1 6}$ showed characteristic triplet and quartet signals at $\delta 1.38$ and $4.36 \mathrm{ppm}$, respectively, assigned to the ethoxy protons.

On the other hand, Friedländer condensation of $o$-aminoaldehyde 12 with active methylene compounds containing the $-\mathrm{COCH}_{2} \mathrm{CO}-$ moiety were studied to construct some new chromeno[2,3-b]pyridines 18-20. Thus, treatment of $\mathbf{1 2}$ with ethyl acetoacetate and ethyl 
benzoylacetate in ethanol containing DBU gave heteroannulated chromones, ethyl 9-allyl-2methyl-5-oxo-5H-chromeno[2,3-b]pyridine-3-carboxylate 18 and its corresponding 2-phenyl analog 19, respectively (Scheme 5). IR spectra of compounds 18 and 19 showed characteristic absorption bands at $1728 / 1712$ and $1673 / 1668 \mathrm{~cm}^{-1}$ for $\left(\mathrm{C}=\mathrm{O}_{\text {ester }}\right)$ and $\left(\mathrm{C}=\mathrm{O}_{\gamma \text {-pyrone }}\right)$, respectively. The ${ }^{1} \mathrm{H}$ NMR spectrum of $\mathbf{1 8}$ showed triplet and quartet signals at $\delta 1.40$ and $4.39 \mathrm{ppm}$, respectively, in addition to characteristic singlet signals at $\delta 2.82$ and $8.88 \mathrm{ppm}$ due to the $\mathrm{CH}_{3}$ protons in position 2 and $\mathrm{H}-4$, respectively.

Also, Friedländer condensation of $\mathbf{1 2}$ with barbituric acid in the presence of DBU furnished 10-allylchromeno[2',3':2,3]pyrido[6,5- $d$ pyrimidine-2,4,6-trione 20. The IR spectrum of 20 showed absorption bands at $3198(\mathrm{NH}), 1739,1676\left(2 \mathrm{C}=\mathrm{O}_{\text {cyclic amide }}\right)$ and $1650\left(\mathrm{C}=\mathrm{O}_{\gamma \text {-pyrone }}\right)$ $\mathrm{cm}^{-1}$. The ${ }^{1} \mathrm{H}$ NMR spectrum revealed two exchangeable signals at $\delta 11.79$ and $12.20 \mathrm{ppm}$ assigned to $2 \mathrm{NH}$, in addition to a singlet signal at $\delta 8.85$ ppm attributed to H-5.

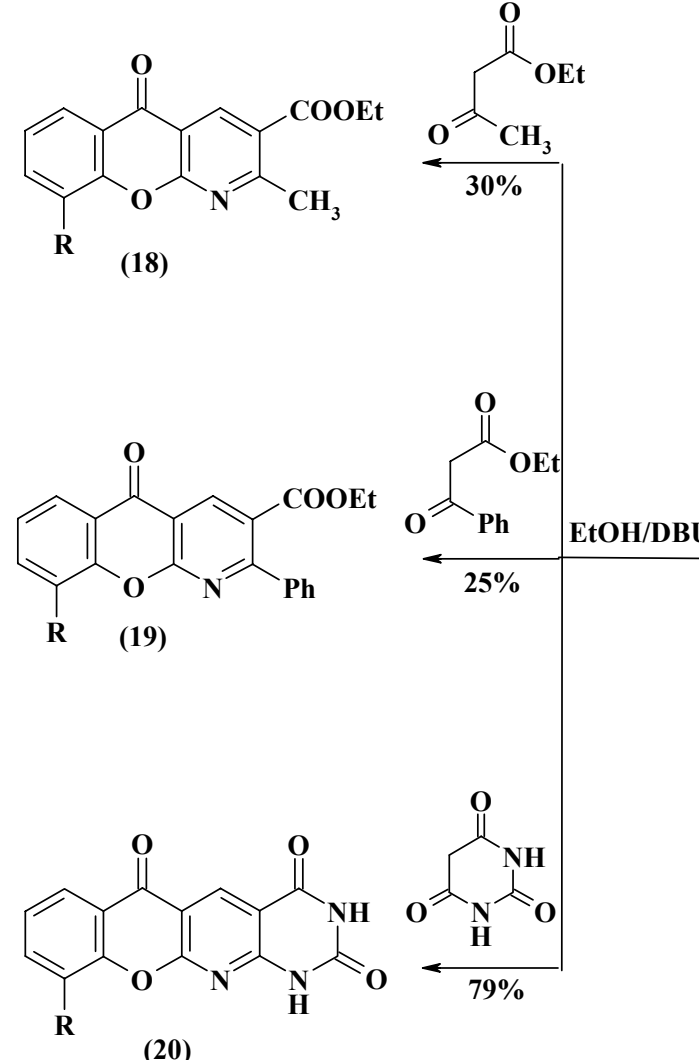

$\mathbf{R}=$
(12)

EtOH/DBU

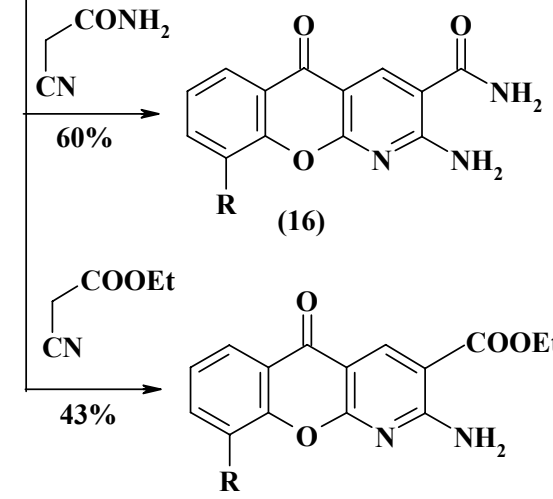

(17)

Scheme 5. Formation of heteroannulated chromones 14-20. 


\section{Experimental Section}

General. All melting points are uncorrected and were recorded in open capillary tubes on a Gallenkamp 595-MFB melting point apparatus. The IR spectra were recorded on an FTIR Bruker Vector 22 spectrophotometer using $\mathrm{KBr}$ wafer technique. ${ }^{1} \mathrm{H}$ NMR spectra were measured on a Varian Gemini spectrophotometer $200 \mathrm{MHz}$ using DMSO- $d_{6}$ as solvent and TMS $(\delta \mathrm{ppm})$ as an internal standard. Mass spectra were obtained using a GCMS qp 1000 ex Schimadzu instrument $(70 \mathrm{eV})$. Elemental microanalyses were performed at the Cairo University Microanalytical Center.

8-Allylchromone-3-carboxaldehyde 4. Phosphoryl chloride (14 mL, $153 \mathrm{mmol})$ was added drop wise to a pre-cooled DMF $(37.5 \mathrm{~mL}, 500 \mathrm{mmol})$ and the mixture was stirred at room temperature for $30 \mathrm{~min}$. Then 3-allyl-2-hydroxyacetophenone $(7 \mathrm{~mL}, 39.8 \mathrm{mmol})$ was added drop wise with continuous stirring. The mixture was stirred at room temperature for $2 \mathrm{~h}$, left overnight and poured into crushed ice $(50 \mathrm{~g})$. The solid obtained was filtered, dried in air and crystallized from petroleum ether (60-80) to give 4 as yellow crystals, yield $3.5 \mathrm{~g}$ (42\%), m.p. 73-74 ${ }^{\circ} \mathrm{C}$. IR $\left(\mathrm{KBr}, \mathrm{cm}^{-1}\right): 1700\left(\mathrm{C}=\mathrm{O}_{\text {aldehyde }}\right), 1647\left(\mathrm{C}=\mathrm{O}_{\gamma \text {-pyrone }}\right)$ and $1607(\mathrm{C}=\mathrm{C}) \mathrm{cm}^{-1} .{ }^{1} \mathrm{H}$ NMR $\left(\mathrm{DMSO}_{6}, \delta\right): 3.62\left(2 \mathrm{H}, \mathrm{d}, J=6.4 \mathrm{~Hz}, \mathrm{H}-1^{\prime}\right), 5.06$ (2H, m, H-3'), 6.05 (1H, m, H-2'), 7.50 (1H, t, $J=7.4 \mathrm{~Hz}, \mathrm{H}-6), 7.73(1 \mathrm{H}, \mathrm{dd}, J=7.6$ and $1.2 \mathrm{~Hz}, \mathrm{H}-7), 8.02(1 \mathrm{H}, \mathrm{dd}, J=7.9$ and $1.8 \mathrm{~Hz}, \mathrm{H}-5)$, $8.93(1 \mathrm{H}, \mathrm{s}, \mathrm{H}-2), 10.12(1 \mathrm{H}, \mathrm{s}, \mathrm{CHO})$. Anal. Calcd. for $\mathrm{C}_{13} \mathrm{H}_{10} \mathrm{O}_{3}$ (214.22): C, 72.89; H, 4.71. Found C, 72.90; H, 4.80.

4-(8-Allylchromon-3-ylmethylene)-2-phenyl-4H-oxazol-5-one 5. To a mixture of hippuric acid $(0.358 \mathrm{~g}, 2 \mathrm{mmol})$ and freshly fused sodium acetate $(0.4 \mathrm{~g})$ in $\mathrm{Ac}_{2} \mathrm{O}(20 \mathrm{~mL})$, compound $4(0.428$ $\mathrm{g}, 2 \mathrm{mmol}$ ) was added. The reaction mixture was heated at reflux for $30 \mathrm{~min}$. The solid deposited after cooling was filtered and recrystallized from ethanol to give $\mathbf{5}$ as yellow crystals, yield 0.43 g (60\%), m.p. $177-178{ }^{\circ} \mathrm{C}$. IR ( $\left.\mathrm{KBr}, \quad \mathrm{cm}^{-1}\right): 1803\left(\mathrm{C}=\mathrm{O}_{\text {oxazolone }}\right), 1657\left(\mathrm{C}=\mathrm{O}_{\gamma \text {-pyrone }}\right), 1611$ and $1568(\mathrm{C}=\mathrm{N}$ and $\mathrm{C}=\mathrm{C}) \mathrm{cm}^{-1} .{ }^{1} \mathrm{H}$ NMR $\left(\mathrm{DMSO}-d_{6}, \delta\right): 3.67(2 \mathrm{H}, \mathrm{d}, J=6.2 \mathrm{~Hz}, \mathrm{H}-1 "), 5.18(2 \mathrm{H}, \mathrm{m}$, H-3"), 6.20 (1H, m, H-2"), 7.37-8.17 (8H, m, Ar-H), $8.21\left(1 \mathrm{H}, \mathrm{s}, \mathrm{H}-2^{\prime}\right)$ and $9.75(1 \mathrm{H}, \mathrm{s}, \mathrm{CH}=\mathrm{C})$. Anal. Calcd. for $\mathrm{C}_{22} \mathrm{H}_{15} \mathrm{NO}_{4}$ (357.37): C, 73.94; H, 4.23; N, 3.92. Found C, 74.01; H, 3.97; N, 3.91 .

5-(8-Allylchromon-3-ylmethylene)-1,3-dihydropyrimidine-2,4,6-trione 6. To a solution of 4 $(0.428 \mathrm{~g}, 2 \mathrm{mmol})$ in dry pyridine $(5 \mathrm{~mL})$, a solution of barbituric acid $(0.3 \mathrm{~g}, 2.5 \mathrm{mmol})$ in dry pyridine $(5 \mathrm{~mL})$ was added. The reaction mixture was heated on a water bath for $1 \mathrm{~h}$. The mixture was cooled and the solid deposited after acidification with dil. $\mathrm{HCl}$ was filtered and crystallized from aqueous dioxane to give 6 as yellow crystals, yield $0.42 \mathrm{~g} \mathrm{(65 \% ),} \mathrm{m.p.} \mathrm{186-187}$ ${ }^{\circ} \mathrm{C}$. IR $\left(\mathrm{KBr}, \mathrm{cm}^{-1}\right): 3199(2 \mathrm{NH}), 1747,1704\left(\mathrm{C}=\mathrm{O}_{\text {cyclic amide }}\right), 1673\left(\mathrm{C}=\mathrm{O}_{\gamma \text {-pyrone }}\right)$ and $1589(\mathrm{C}=\mathrm{C})$ $\mathrm{cm}^{-1} .{ }^{1} \mathrm{H}$ NMR (DMSO- $d_{6}, \delta$ ): 3.66 (2H, d, $\left.J=6.4 \mathrm{~Hz}, \mathrm{H}-1 "\right), 5.16$ (2H, m, H-3"), 6.08 (1H, m, H-2"), $7.56\left(1 \mathrm{H}, \mathrm{t}, J=7.6 \mathrm{~Hz}, \mathrm{H}-6^{\prime}\right), 7.75\left(1 \mathrm{H}, \mathrm{dd}, J=7.4\right.$ and $\left.1.6 \mathrm{~Hz}, \mathrm{H}-7^{\prime}\right), 8.05(1 \mathrm{H}, \mathrm{dd}, J=$ 7.8 and $\left.1.7 \mathrm{~Hz}, \mathrm{H}-5^{\prime}\right), 8.44\left(1 \mathrm{H}, \mathrm{s}, \mathrm{H}-2^{\prime}\right), 9.77(1 \mathrm{H}, \mathrm{s}, \mathrm{CH}=\mathrm{C}), 11.37$ and $11.47(2 \mathrm{H}$, each s, 2NH 
exchangeable with $\mathrm{D}_{2} \mathrm{O}$ ). Anal. Calcd. for $\mathrm{C}_{17} \mathrm{H}_{12} \mathrm{~N}_{2} \mathrm{O}_{5}$ (324.30): C, 62.96; H, 3.73; N, 8.64. Found C, 62.95; H, 3.85; N, 8.63.

3,3,6,6-Tetramethyl-9-(8-allylchromon-3-yl)-1,2,3,4,5,6,7,8-octahydroxanthene-1,8-dione 7. To a solution of $4(0.428 \mathrm{~g}, 2 \mathrm{mmol})$ in dry pyridine $(5 \mathrm{~mL})$, dimedone $(0.8 \mathrm{~g}, 5.7 \mathrm{mmol})$ was added and stirred at room temperature for $1 \mathrm{~h}$, then the mixture was acidified with $6 \mathrm{~N} \mathrm{HCl}$. The solid deposited was filtered and crystallized from aqueous dioxane to give 7 as white crystals, yield 0.8 g (90\%), m.p. $200-201{ }^{\circ} \mathrm{C}$. IR $\left(\mathrm{KBr}, \mathrm{cm}^{-1}\right): 1662\left(\mathrm{C}=\mathrm{O}_{\text {xanthene }}\right), 1632\left(\mathrm{C}=\mathrm{O}_{\gamma \text {-pyrone }}\right)$ and $1579(\mathrm{C}=\mathrm{C}) \mathrm{cm}^{-1} .{ }^{1} \mathrm{H}$ NMR (DMSO- $\left.d_{6}, \delta\right): 0.87\left(6 \mathrm{H}, \mathrm{s}, 2 \mathrm{CH}_{3}\right), 1.02\left(6 \mathrm{H}, \mathrm{s}, 2 \mathrm{CH}_{3}\right), 2.01-2.61$ $\left(8 \mathrm{H}, \mathrm{m}, 4 \mathrm{CH}_{2}\right), 3.57$ (2H, d, $\left.J=5.5 \mathrm{~Hz}, \mathrm{H}-1 "\right), 4.34$ (1H, s, pyran H), 5.16 (2H, m, H-3"), 5.98 (1H, m, H-2"), 7.33 (1H, m, H-6'), 7.59 (1H, d, J= 7.2 Hz, H-7'), 7.87 (1H, d, J= 7.8 Hz, H-5') and $8.35\left(1 \mathrm{H}, \mathrm{s}, \quad \mathrm{H}-2^{\prime}\right)$. Anal. Calcd. for $\mathrm{C}_{29} \mathrm{H}_{30} \mathrm{O}_{5}$ (458.56): C, 75.96; H, 6.59. Found C, 75.62; $\mathrm{H}, 6.44$.

8-Allylchromone-3-carboxaldehyde-oxime 8. A mixture of 4 (2.14 g, $10 \mathrm{mmol})$ in ethanol (15 $\mathrm{mL})$ and hydroxylamine hydrochloride $(0.77 \mathrm{~g}, 11 \mathrm{mmol})$ was heated at reflux for $15 \mathrm{~min}$. The solid obtained after cooling was filtered and recrystallized from ethanol to give $\mathbf{8}$ as white crystals, yield $2.15 \mathrm{~g}(94 \%)$, m.p. $163-164{ }^{\circ} \mathrm{C}$. IR (KBr, cm $\left.{ }^{-1}\right): 3267(\mathrm{OH}), 1636\left(\mathrm{C}=\mathrm{O}_{\gamma \text {-pyrone }}\right)$ 1613 and $1574(\mathrm{C}=\mathrm{N}$ and $\mathrm{C}=\mathrm{C}) \mathrm{cm}^{-1} .{ }^{1} \mathrm{H}$ NMR $\left(\mathrm{DMSO}-d_{6}, \delta\right): 3.65\left(2 \mathrm{H}, \mathrm{d}, J=6.2 \mathrm{~Hz}, \mathrm{H}-1^{\prime}\right)$, 5.14 (2H, m, H-3'), 6.00 (1H, m, H-2'), 7.44 (1H, m, H-6), 7.70 (1H, d, J=7.0, H-7), 8.00 (1H, $\mathrm{dd}, J=8.8$ and $1.8 \mathrm{~Hz}, \mathrm{H}-5), 8.09(1 \mathrm{H}, \mathrm{s}, \mathrm{CH}=\mathrm{N}), 8.72(1 \mathrm{H}, \mathrm{s}, \mathrm{H}-2), 11.44\left(1 \mathrm{H}, \mathrm{s}, \mathrm{OH}_{\text {oxime}}\right)$. Anal. Calcd. for $\mathrm{C}_{13} \mathrm{H}_{11} \mathrm{NO}_{3}$ (229.24): C, 68.11; H, 4.84; N, 6.11. Found C, 67.95; H, 4.90; N, 6.14 .

8-Allylchromone-3-carbonitrile 9. A mixture of $8(0.45 \mathrm{~g}, 2 \mathrm{mmol})$ and $\mathrm{Ac}_{2} \mathrm{O}(2 \mathrm{~mL})$ was heated at reflux for $4 \mathrm{~h}$. The reaction mixture was cooled and poured into crushed ice $(30 \mathrm{~g})$; the solid obtained was filtered and recrystallized from benzene/petroleum ether (40-60) to give 9 as yellow crystals, yield 0.34 g $(82 \%)$, m.p. $97-98{ }^{\circ} \mathrm{C}$. IR $\left(\mathrm{KBr}, \mathrm{cm}^{-1}\right): 2239(\mathrm{C} \equiv \mathrm{N}), 1658\left(\mathrm{C}=\mathrm{O}_{\gamma \text { - }}\right.$ pyrone) and $1616(\mathrm{C}=\mathrm{C}) \mathrm{cm}^{-1} .{ }^{1} \mathrm{H}$ NMR $\left(\mathrm{DMSO}_{-}, \delta\right): 3.65\left(2 \mathrm{H}, \mathrm{d}, J=6.0 \mathrm{~Hz}, \mathrm{H}-1^{\prime}\right), 5.07(2 \mathrm{H}, \mathrm{m}$, H-3'), 6.00 (1H, m, H-2'), 7.55 (1H, t, $J=7.8 \mathrm{~Hz}, \mathrm{H}-6), 7.80(1 \mathrm{H}, \mathrm{d}, J=7.4 \mathrm{~Hz}, \mathrm{H}-7), 8.01(1 \mathrm{H}$, $\mathrm{d}, J=7.8 \mathrm{~Hz}, \mathrm{H}-5), 9.28(1 \mathrm{H}, \mathrm{s}, \mathrm{H}-2)$. Anal. Calcd. for $\mathrm{C}_{13} \mathrm{H}_{9} \mathrm{NO}_{2}(211.22): \mathrm{C}, 73.92 ; \mathrm{H}, 4.29 ; \mathrm{N}$, 6.63. Found C, 73.26; H, 4.04; N, 6.84 .

8-Allyl-3-(1H-tetrazol-5-yl)chromone 10. To THF $(8 \mathrm{ml})$ pre-cooled in an ice-bath were added pulverized anhydrous $\mathrm{AlCl}_{3}(0.585 \mathrm{~g}, 4.4 \mathrm{mmol})$, sodium azide $(0.572 \mathrm{~g}, 8.8 \mathrm{mmol})$ and compound 9 ( $0.422 \mathrm{~g}, 2 \mathrm{mmol})$. The ice-bath was removed and the mixture was stirred under reflux for $10 \mathrm{~h}$, then left to cool and acidified with $15 \% \mathrm{HCl}(5 \mathrm{~mL})$. The solid obtained was filtered and crystallized from aqueous dioxane to give $\mathbf{1 0}$ as white crystals, yield $0.38 \mathrm{~g}(76 \%)$, m.p. $267-268{ }^{\circ} \mathrm{C}$. IR $\left(\mathrm{KBr}, \mathrm{cm}^{-1}\right): 3192(\mathrm{NH}), 1644\left(\mathrm{C}=\mathrm{O}_{\gamma \text {-pyrone }}\right)$ and $1582(\mathrm{C}=\mathrm{C}) \mathrm{cm}^{-1} .{ }^{1} \mathrm{H} \mathrm{NMR}$ $\left(\mathrm{DMSO}_{6}, \delta\right): 3.68\left(2 \mathrm{H}, \mathrm{d}, J=6.4 \mathrm{~Hz}, \mathrm{H}-1^{\prime}\right), 5.11\left(2 \mathrm{H}, \mathrm{m}, \mathrm{H}-3^{\prime}\right), 6.10(1 \mathrm{H}, \mathrm{m}, \mathrm{H}-2 '), 7.54(1 \mathrm{H}$, m, H-6), 7.61 (1H, d, $J=7.3 \mathrm{~Hz}, \mathrm{H}-7), 8.09$ (1H, d, $J=7.9 \mathrm{~Hz}, \mathrm{H}-5), 9.31$ (1H, s, H-2), 16.58 $\left(1 \mathrm{H}, \mathrm{s}, \mathrm{NH}\right.$ exchangeable with $\left.\mathrm{D}_{2} \mathrm{O}\right)$. Anal. Calcd. for $\mathrm{C}_{13} \mathrm{H}_{10} \mathrm{~N}_{4} \mathrm{O}_{2}(254.25)$ : C, 61.41; $\mathrm{H}, 3.96$; N, 22.04. Found C, 61.70; H, 3.90; N, 21.86. 
3-(8-Allylchromon-3-yl)acrylic acid 11. To a solution of $4(0.32 \mathrm{~g}, 15 \mathrm{mmol})$ in dry pyridine (5 $\mathrm{mL})$, malonic acid $(0.26 \mathrm{~g}, 25 \mathrm{mmol})$ was added. The reaction mixture was heated on a water bath for $2 \mathrm{~h}$. The solid deposited after acidification with dil. $\mathrm{HCl}$ was filtered and crystallized from acetic acid to give 11 as yellow crystals, yield $0.32 \mathrm{~g}(85 \%)$, m.p. $195-196{ }^{\circ} \mathrm{C}$. IR $(\mathrm{KBr}$, $\left.\mathrm{cm}^{-1}\right)$ : 3200-2500 (OH), $1701\left(\mathrm{C}=\mathrm{O}_{\text {carboxy }}\right), 1652\left(\mathrm{C}=\mathrm{O}_{\gamma \text {-pyrone }}\right)$ and $1614(\mathrm{C}=\mathrm{C}) \mathrm{cm}^{-1} .{ }^{1} \mathrm{H}$ NMR $\left(\mathrm{DMSO}_{6}, \delta\right): 3.50\left(2 \mathrm{H}, \mathrm{d}, J=6.4 \mathrm{~Hz}, \mathrm{H}-1^{\prime \prime}\right), 5.09(2 \mathrm{H}, \mathrm{m}, \mathrm{H}-3 "), 6.02(1 \mathrm{H}, \mathrm{m}, \mathrm{H}-2 "), 7.13$ $(1 \mathrm{H}, \mathrm{d}, J=15.8 \mathrm{~Hz}$, olefinic proton), $7.43(2 \mathrm{H}, \mathrm{m}, \mathrm{H}-6$ ' + olefinic $\mathrm{H}), 7.67(1 \mathrm{H}, \mathrm{d}, J=7.0 \mathrm{~Hz}, \mathrm{H}-$ $\left.7^{\prime}\right), 8.20\left(1 \mathrm{H}, \mathrm{d}, J=6.5 \mathrm{~Hz}, \mathrm{H}-5^{\prime}\right), 8.88\left(1 \mathrm{H}, \mathrm{s}, \mathrm{H}-2^{\prime}\right)$ and $12.41(1 \mathrm{H}, \mathrm{s}, \mathrm{COOH}$ exchangeable with $\mathrm{D}_{2} \mathrm{O}$ ). Anal. Calcd. for $\mathrm{C}_{15} \mathrm{H}_{12} \mathrm{O}_{4}$ (256.26): C, 70.31; H, 4.72. Found C, 70.46; H, 4.58.

\section{8-Allyl-2-aminochromone-3-carboxaldehyde 12}

a) Using $\mathbf{N a O H}$. A mixture of $8(2.29 \mathrm{~g}, 10 \mathrm{mmol})$ or $9(2.11,10 \mathrm{mmol})$ and $0.05 \mathrm{M}$ sodium hydroxide solution $(15 \mathrm{~mL})$ was stirred at $70^{\circ} \mathrm{C}$ for $2 \mathrm{~h}$. Water was added $(50 \mathrm{~mL})$ and the solid obtained was filtered and recrystallized from ethanol to give $\mathbf{1 2}$ as yellow crystals, yield (70\%), m.p. $225-226^{\circ} \mathrm{C}$.

b) Using $\mathrm{NH}_{4} \mathrm{OH}$. A mixture of $8(2.29 \mathrm{~g}, 10 \mathrm{mmol})$ or $9(2.11,10 \mathrm{mmol})$ and concentrated $\mathrm{NH}_{4} \mathrm{OH}$ solution $(10 \mathrm{~mL})$ was stirred at room temperature until the solid dissolved and then was diluted with water $(10 \mathrm{~mL})$. The product obtained was filtered and recrystallized from ethanol to give 12 as yellow crystals, yield $(90 \%)$, m.p. $225-226{ }^{\circ} \mathrm{C}$. IR $\left(\mathrm{KBr}, \mathrm{cm}^{-1}\right)$ : 3304, $3175\left(\mathrm{NH}_{2}\right)$, $1665\left(\mathrm{C}=\mathrm{O}_{\text {aldehyde }}\right), 1635\left(\mathrm{C}=\mathrm{O}_{\gamma \text {-pyrone }}\right)$ and $1602(\mathrm{C}=\mathrm{C}) \mathrm{cm}^{-1} .{ }^{1} \mathrm{H}$ NMR (DMSO- $\left.d_{6}, \delta\right): 3.62(2 \mathrm{H}$, d, $\left.J=6.5 \mathrm{~Hz}, \mathrm{H}-1^{\prime}\right), 5.21\left(2 \mathrm{H}, \mathrm{m}, \mathrm{H}-3^{\prime}\right), 6.00\left(1 \mathrm{H}, \mathrm{m}, \mathrm{H}-2^{\prime}\right), 7.42(1 \mathrm{H}, \mathrm{m}, \mathrm{H}-6), 7.62(1 \mathrm{H}, \mathrm{d}, J=$ $7.3 \mathrm{~Hz}, \mathrm{H}-7), 7.94(1 \mathrm{H}, \mathrm{dd}, J=7.6$ and $1.7 \mathrm{~Hz}, \mathrm{H}-5), 9.62\left(2 \mathrm{H}, \mathrm{s}, \mathrm{NH}_{2}\right), 10.13(1 \mathrm{H}, \mathrm{s}, \mathrm{CHO}) . M / e$ (relative intensity): $229\left(\mathrm{M}^{+}, 16\right), 230\left(\mathrm{M}^{+}+1,4\right), 201(100), 200(38), 188$ (2), 172 (4), 131 (58), 116 (13), 103 (21), 77 (45), 76 (10), 68 (34). Anal. Calcd. for $\mathrm{C}_{13} \mathrm{H}_{11} \mathrm{NO}_{3}$ (229.24): C, 68.11; H, 4.84; N, 6.11. Found C, 68.23; H, 4.69; N, 6.18.

8-Allyl-2-aminochromone-3-carboxaldehyde-oxime 13. To a solution of $12(0.458 \mathrm{~g}, 2 \mathrm{mmol})$ in ethanol $(5 \mathrm{~mL})$, hydroxylamine hydrochloride $(0.154 \mathrm{~g}, 2.2 \mathrm{mmol})$ was added. The reaction mixture was heated at reflux on a water bath for $15 \mathrm{~min}$. The solid obtained was filtered and recrystallized from ethanol to give $\mathbf{1 3}$ as white crystals, yield $0.3 \mathrm{~g}(63 \%)$, m.p. $231-232{ }^{\circ} \mathrm{C}$. IR $\left(\mathrm{KBr}, \mathrm{cm}^{-1}\right): 3355,3214\left(\mathrm{NH}_{2}\right.$ and $\left.\mathrm{OH}\right), 1644\left(\mathrm{C}=\mathrm{O}_{\gamma \text {-pyrone }}\right), 1604$ and $1544(\mathrm{C}=\mathrm{N}$ and $\mathrm{C}=\mathrm{C}) \mathrm{cm}^{-1}$. ${ }^{1} \mathrm{H}$ NMR (DMSO- $\left.d_{6}, \delta\right): 3.61\left(2 \mathrm{H}, \mathrm{d}, J=6.7 \mathrm{~Hz}, \mathrm{H}-1^{\prime}\right), 5.14\left(2 \mathrm{H}, \mathrm{m}, \mathrm{H}-3^{\prime}\right), 6.12\left(1 \mathrm{H}, \mathrm{m}, \mathrm{H}-2^{\prime}\right)$, $7.30(1 \mathrm{H}, \mathrm{t}, J=7.5 \mathrm{~Hz}, \mathrm{H}-6), 7.51(1 \mathrm{H}, \mathrm{d}, J=7.8, \mathrm{H}-7), 7.85(1 \mathrm{H}, \mathrm{dd}, J=7.8$ and $1.58 \mathrm{~Hz}, \mathrm{H}-5)$, $8.44(1 \mathrm{H}, \mathrm{s}, \mathrm{CH}=\mathrm{N}), 8.90\left(2 \mathrm{H}, \mathrm{br}, \mathrm{NH}_{2}\right), 10.77\left(1 \mathrm{H}, \mathrm{s}, \mathrm{OH}_{\text {oxime }}\right)$. Anal. Calcd. for $\mathrm{C}_{13} \mathrm{H}_{12} \mathrm{~N}_{2} \mathrm{O}_{3}$ (244.25): C, 63.93; H,4.95; N,11.47. Found C, 63.82; H, 5.03; N, 11.31.

9-Allyl-2-amino-5-oxo-5H-chromeno[2,3-b]pyridine-3-carbonitrile 14 . To a solution of $\mathbf{1 2}$ $(0.458 \mathrm{~g}, 2 \mathrm{mmol})$ in absolute ethanol $(25 \mathrm{~mL})$ and DBU $(0.4 \mathrm{~mL})$, malononitrile $(0.56 \mathrm{~g}, 2$ mmol) was added. The reaction mixture was heated at reflux for $30 \mathrm{~min}$. The solid obtained during heating was filtered and recrystallized from aqueous DMF to give $\mathbf{1 4}$ as yellow crystals, yield 0.37 g (67\%), m.p. $263-264{ }^{\circ} \mathrm{C}$. IR $\left(\mathrm{KBr}, \mathrm{cm}^{-1}\right): 3458,3348\left(\mathrm{NH}_{2}\right), 2221(\mathrm{C} \equiv \mathrm{N}), 1662$

$\left(\mathrm{C}=\mathrm{O}_{\gamma \text {-pyrone }}\right), 1602$ and $1541(\mathrm{C}=\mathrm{N}$ and $\mathrm{C}=\mathrm{C}) \mathrm{cm}^{-1} .{ }^{1} \mathrm{H}$ NMR (DMSO- $\left.d_{6}, \delta\right): 3.60(2 \mathrm{H}, \mathrm{d}, J=5.6$ Hz, H-1'), 5.07 (2H, m, H-3'), 6.04 (1H, m, H-2'), 7.37 (1H, t, $J=7.6 \mathrm{~Hz}, \mathrm{H}-7), 7.62(1 \mathrm{H}, \mathrm{d}, J=$ 
$7.0 \mathrm{~Hz}, \mathrm{H}-8), 7.92(1 \mathrm{H}, \mathrm{d}, J=7.6 \mathrm{~Hz}, \mathrm{H}-6), 8.13\left(2 \mathrm{H}, \mathrm{br}, \mathrm{NH}_{2}\right)$ and $8.54(1 \mathrm{H}, \mathrm{s}, \mathrm{H}-4)$. M/e (relative intensity): $277\left(\mathrm{M}^{+}, 91\right), 278\left(\mathrm{M}^{+}+1,10\right), 250$ (9), 222 (7), 162 (51), 140 (5), 133 (12), 117 (5), 105 (9), 89 (14), 77 (18), 65 (14). Anal. Calcd. for $\mathrm{C}_{16} \mathrm{H}_{11} \mathrm{~N}_{3} \mathrm{O}_{3}$ (277.28): C, 69.31; $\mathrm{H}$, 4.00; N,15.15. Found C, 69.61; H, 4.02; N, 15.02.

9-Allyl-2-amino-3-phenylthio-chromeno[2,3-b]pyridin-5-one 15. A mixture of 12 (0.229 g, 1 $\mathrm{mmol}), \mathrm{DBU}(0.4 \mathrm{~mL})$ and phenylthioacetonitrile $(0.15 \mathrm{~mL}, 1 \mathrm{mmol})$ was heated at reflux in absolute ethanol $(20 \mathrm{~mL})$ for $3 \mathrm{~h}$. The solid obtained after cooling was filtered and recrystallized from ethanol to give 15 as a pale yellow crystals, yield $0.24 \mathrm{~g}(34 \%)$, m.p. $234-235{ }^{\circ} \mathrm{C}$. IR $(\mathrm{KBr}$, $\left.\mathrm{cm}^{-1}\right)$ : 3456, $3266\left(\mathrm{NH}_{2}\right), 1660\left(\mathrm{C}=\mathrm{O}_{\gamma \text {-pyrone }}\right) 1625$ and $1579(\mathrm{C}=\mathrm{N}$ and $\mathrm{C}=\mathrm{C}) \mathrm{cm}^{-1} .{ }^{1} \mathrm{H}$ NMR $\left(\mathrm{DMSO}_{6}, \delta\right): 3.67\left(2 \mathrm{H}, \mathrm{d}, J=6.3 \mathrm{~Hz}, \mathrm{H}-1^{\prime}\right), 5.10\left(2 \mathrm{H}, \mathrm{m}, \mathrm{H}-3^{\prime}\right), 6.10\left(1 \mathrm{H}, \mathrm{m}, \mathrm{H}-2^{\prime}\right), 7.25-8.02$ $(8 \mathrm{H}, \mathrm{m}, \mathrm{Ar}-\mathrm{H}), 7.98\left(2 \mathrm{H}, \mathrm{br}, \mathrm{NH}_{2}\right)$ and $8.36(1 \mathrm{H}, \mathrm{s}, \mathrm{H}-4)$. Anal. Calcd. for $\mathrm{C}_{21} \mathrm{H}_{16} \mathrm{~N}_{2} \mathrm{O}_{2} \mathrm{~S}$ (360.44): C, 69.98; H, 4.47; N,7.77; S, 8.88. Found C, 70.34; H, 4.43; N, 7.90; S, 8.88.

9-Allyl-2-amino-5-oxo-5 $H$-chromeno[2,3-b]pyridine-3-carboxamide 16. A mixture of 12 $(0.229 \mathrm{~g}, 1 \mathrm{mmol})$, DBU $(0.4 \mathrm{~mL})$ and cyanoacetamide $(0.84 \mathrm{~g}, 1 \mathrm{mmol})$ was heated at reflux in absolute ethanol $(20 \mathrm{~mL})$ for $30 \mathrm{~min}$. The solid obtained during heating was filtered and recrystallized from aqueous DMF to give 16 as yellow crystals, yield $0.177 \mathrm{~g}(60 \%)$, m.p. above $300{ }^{\circ} \mathrm{C}$. IR $\left(\mathrm{KBr}, \mathrm{cm}^{-1}\right): 3389,3228,3173\left(2 \mathrm{NH}_{2}\right), 1653\left(\mathrm{C}=\mathrm{O}_{\text {amide }}\right), 1624\left(\mathrm{C}=\mathrm{O}_{\gamma \text {-pyrone }}\right) 1604$ and $1545(\mathrm{C}=\mathrm{N}$ and $\mathrm{C}=\mathrm{C}) \mathrm{cm}^{-1} .{ }^{1} \mathrm{H}$ NMR $\left(\mathrm{DMSO}-d_{6}, \delta\right): 3.64\left(2 \mathrm{H}, \mathrm{d}, J=5.2 \mathrm{~Hz}, \mathrm{H}-1^{\prime}\right), 5.08(2 \mathrm{H}, \mathrm{m}$, H-3'), 6.05 (1H, m, H-2'), 7.39 (1H, t, $J=7.6 \mathrm{~Hz}, \mathrm{H}-7), 7.53\left(2 \mathrm{H}, \mathrm{br}, \mathrm{NH}_{2}\right), 7.67(1 \mathrm{H}, \mathrm{d}, J=7.02$ $\mathrm{Hz}, \mathrm{H}-8), 8.02(1 \mathrm{H}, \mathrm{d}, J=7.32 \mathrm{~Hz}, \mathrm{H}-6), 8.41\left(2 \mathrm{H}, \mathrm{br}, \mathrm{CONH}_{2}\right)$ and $8.81(1 \mathrm{H}, \mathrm{s}, \mathrm{H}-4) . M / e$ (relative intensity): $295\left(\mathrm{M}^{+}, 100\right), 296\left(\mathrm{M}^{+}+1,18\right), 278$ (10), 250 (23), 223 (5), 163 (8), 133 (12), 115 (17), 94 (14), 77 (12), 67 (14). Anal. Calcd. for $\mathrm{C}_{16} \mathrm{H}_{13} \mathrm{~N}_{3} \mathrm{O}_{3}$ (295.30): C, 65.08; H, $4.44 ; \mathrm{N}, 14.23$. Found C, 64.68; H, 4.56; N, 13.83 .

Ethyl 9-allyl-2-amino-5-oxo-5H-chromeno[2,3-b]pyridine-3-carboxylate 17. To a solution of $12(0.458 \mathrm{~g}, 2 \mathrm{mmol})$ in absolute ethanol $(20 \mathrm{~mL})$ and DBU $(0.4 \mathrm{~mL})$, ethyl cyanoacetate $(0.226$ $\mathrm{g}, 2 \mathrm{mmol}$ ) was added. The reaction mixture was heated at reflux for $30 \mathrm{~min}$, then left to cool at room temperature. The solid obtained was filtered and recrystallized from ethanol to give $\mathbf{1 7}$ as white crystals, yield 0.28 g (43\%), m.p. $239-240{ }^{\circ} \mathrm{C}$. IR $\left(\mathrm{KBr}, \mathrm{cm}^{-1}\right): 3407,3272\left(\mathrm{NH}_{2}\right)$, 1697( $\left.\mathrm{C}=\mathrm{O}_{\text {ester }}\right), 1667\left(\mathrm{C}=\mathrm{O}_{\gamma \text {-pyrone }}\right) 1626$ and $1593(\mathrm{C}=\mathrm{N}$ and $\mathrm{C}=\mathrm{C}) \mathrm{cm}^{-1} .{ }^{1} \mathrm{H}$ NMR $\left(\mathrm{DMSO}-d_{6}, \delta\right)$ : $1.38\left(3 \mathrm{H}, \mathrm{t}, J=7.1 \mathrm{~Hz}, \mathrm{OCH}_{2} \mathrm{CH}_{3}\right), 3.64(2 \mathrm{H}, \mathrm{d}, J=6.9 \mathrm{~Hz}, \mathrm{H}-1$ '), $4.38(2 \mathrm{H}, \mathrm{q}, J=7.2 \mathrm{~Hz}$, $\left.\mathrm{OCH}_{2} \mathrm{CH}_{3}\right), 5.09$ (2H, m, H-3'), $6.12\left(1 \mathrm{H}, \mathrm{m}, \mathrm{H}-2^{\prime}\right), 7.41(1 \mathrm{H}, \mathrm{t}, J=7.6 \mathrm{~Hz}, \mathrm{H}-7), 7.68(1 \mathrm{H}, \mathrm{d}, J=$ $7.2 \mathrm{~Hz}, \mathrm{H}-8), 8.01(1 \mathrm{H}, \mathrm{dd}, J=7.2$ and $1.9 \mathrm{~Hz}, \mathrm{H}-6), 8.10$ and $8.49(2 \mathrm{H}$, each s, $2 \mathrm{NH}$ exchangeable with $\left.\mathrm{D}_{2} \mathrm{O}\right), 8.81(1 \mathrm{H}, \mathrm{s}, \mathrm{H}-4)$. M/e (relative intensity): $324\left(\mathrm{M}^{+}, 52\right), 325\left(\mathrm{M}^{+}+1\right.$, 10), 277 (8), 250 (12), 178 (24), 152 (10), 128 (25), 99 (33), 97 (81), 77 (10), 69 (100), 55 (78). Anal. Calcd for $\mathrm{C}_{18} \mathrm{H}_{16} \mathrm{~N}_{2} \mathrm{O}_{4}$ (324.34): C, 66.66; H, 4.97; N, 8.64. Found C, 66.65; H, 4.84; N, 8.62 .

Ethyl 9-allyl-2-methyl-5-oxo-5H-chromeno[2,3-b]pyridine-3-carboxylate 18. A mixture of 12 $(0.29 \mathrm{~g}, 1.2 \mathrm{mmol})$, DBU $(0.4 \mathrm{~mL})$ and ethyl acetoacetate $(0.17 \mathrm{~mL}, 1.3 \mathrm{mmol})$ was heated at reflux in absolute ethanol $(20 \mathrm{~mL})$ for $6 \mathrm{~h}$. The solid so formed after cooling was filtered and recrystallized from ethanol to give $\mathbf{1 8}$ as white crystals, yield $0.12 \mathrm{~g} \mathrm{(30 \% ),} \mathrm{m.p.} 128-129{ }^{\circ} \mathrm{C}$. IR 
$\left(\mathrm{KBr}, \mathrm{cm}^{-1}\right): 1728\left(\mathrm{C}=\mathrm{O}_{\text {ester }}\right), 1673\left(\mathrm{C}=\mathrm{O}_{\gamma \text {-pyrone }}\right) 1599$ and $1552(\mathrm{C}=\mathrm{N}$ and $\mathrm{C}=\mathrm{C}) \mathrm{cm}^{-1} .{ }^{1} \mathrm{H}$ NMR $\left(\mathrm{DMSO}-d_{6}, \delta\right): 1.40\left(3 \mathrm{H}, \mathrm{t}, J=7.2 \mathrm{~Hz}, \mathrm{OCH}_{2} \mathrm{CH}_{3}\right), 2.82\left(3 \mathrm{H}, \mathrm{s}, \mathrm{CH}_{3}\right), 3.72(2 \mathrm{H}, \mathrm{d}, J=6.1 \mathrm{~Hz}$, H-1'), 4.39 (2H, q, $\left.J=7.3 \mathrm{~Hz}, \mathrm{OCH}_{2} \mathrm{CH}_{3}\right), 5.18(2 \mathrm{H}, \mathrm{m}, \mathrm{H}-3$ '), 6.11 (1H, m, H-2'), 7.48 (1H, t, $J$ $=7.94 \mathrm{~Hz}, \mathrm{H}-7), 7.79(1 \mathrm{H}, \mathrm{d}, J=7.1 \mathrm{~Hz}, \mathrm{H}-8), 8.06(1 \mathrm{H}, \mathrm{d}, J=7.8 \mathrm{~Hz}, \mathrm{H}-6)$ and $8.88(1 \mathrm{H}, \mathrm{s}, \mathrm{H}-$ 4). Anal. Calcd. for $\mathrm{C}_{19} \mathrm{H}_{17} \mathrm{NO}_{4}$ (323.35): C, 70.58; H, 5.30; N, 4.33. Found C, 70.88; H, 4.93; N, 4.37 .

Ethyl 9-allyl-2-phenyl-5-oxo-5H-chromono[2,3-b]pyridine-3-carboxylate 19. A mixture of 12 $(0.229 \mathrm{~g}, 1 \mathrm{mmol})$, DBU $(0.4 \mathrm{ml})$ and ethyl benzoylacetate $(0.2 \mathrm{~mL}, 1 \mathrm{mmol})$ was heated at reflux in absolute ethanol $(20 \mathrm{~mL})$ for $6 \mathrm{~h}$. The reaction mixture was cooled and the solvent was concentrated. The solid obtained was filtered and recrystallized from ethanol to give $\mathbf{1 9}$ as yellow crystals, yield $0.08 \mathrm{~g}(25 \%)$, m.p. $145-146{ }^{\circ} \mathrm{C}$. IR $\left(\mathrm{KBr}, \mathrm{cm}^{-1}\right): 1712\left(\mathrm{C}=\mathrm{O}_{\text {ester }}\right), 1668$ $\left(\mathrm{C}=\mathrm{O}_{\gamma \text {-pyrone }}\right), 1594$ and $1546(\mathrm{C}=\mathrm{N}$ and $\mathrm{C}=\mathrm{C}) \mathrm{cm}^{-1} .{ }^{1} \mathrm{H}$ NMR (DMSO- $\left.d_{6}, \delta\right): 1.37(3 \mathrm{H}, \mathrm{t}, J=7.4$ $\left.\mathrm{Hz}, \mathrm{OCH}_{2} \mathrm{CH}_{3}\right), 3.70\left(2 \mathrm{H}, \mathrm{d}, J=6.1 \mathrm{~Hz}, \mathrm{H}-1^{\prime}\right), 4.21\left(2 \mathrm{H}, \mathrm{q}, J=7.3 \mathrm{~Hz}, \mathrm{OCH}_{2} \mathrm{CH}_{3}\right), 5.25(2 \mathrm{H}, \mathrm{m}$, H-3'), 6.18 (1H, m, H-2'), 7.36-8.12 (7H, m, Ar-H), $8.06(1 \mathrm{H}, \mathrm{d}, J=7.6 \mathrm{~Hz}, \mathrm{H}-6)$ and $8.76(1 \mathrm{H}$, s, H-4). Anal. Calcd. for $\mathrm{C}_{24} \mathrm{H}_{19} \mathrm{NO}_{4}$ (385.42): C, 74.79; H, 4.97; N, 3.63. Found C, 74.70; H, $4.92 ; \mathrm{N}, 3.59$.

10-Allylchromeno[2',3':2,3]pyrido[6,5-d]pyrimidine-2,4,6-trione 20. A mixture of 12 (0.229 $\mathrm{g}, 1 \mathrm{mmol})$, barbituric acid $(0.128 \mathrm{~g}, 1 \mathrm{mmol})$ and DBU $(0.5 \mathrm{~mL})$ was heated at reflux in absolute ethanol $(20 \mathrm{~mL})$ for $30 \mathrm{~min}$. The solid obtained was filtered, dried in air and recrystallized from aqueous DMF to give 20 as white crystals, yield $0.13 \mathrm{~g}(79 \%)$, m.p. above $300^{\circ} \mathrm{C}$. IR $\left(\mathrm{KBr}, \mathrm{cm}^{-1}\right)$ : $3198(\mathrm{NH}), 1739,1676\left(2 \mathrm{C}=\mathrm{O}_{\text {cyclic amide }}\right), 1650\left(\mathrm{C}=\mathrm{O}_{\gamma \text {-pyrone }}\right), 1616$ and $1580(\mathrm{C}=\mathrm{N}$ and $\mathrm{C}=\mathrm{C})$ $\mathrm{cm}^{-1} .{ }^{1} \mathrm{H}$ NMR (DMSO- $\left.d_{6}, \delta\right): 3.68\left(2 \mathrm{H}, \mathrm{d}, J=5.6 \mathrm{~Hz}, \mathrm{H}-1^{\prime}\right), 5.11(2 \mathrm{H}, \mathrm{m}, \mathrm{H}-3$ '), 6.12 (1H, m, H$\left.2^{\prime}\right), 7.48(1 \mathrm{H}, \mathrm{m}, \mathrm{H}-8), 7.76(1 \mathrm{H}, \mathrm{d}, J=7.3 \mathrm{~Hz}, \mathrm{H}-9), 8.05(1 \mathrm{H}, \mathrm{d}, J=7.2 \mathrm{~Hz}, \mathrm{H}-7), 8.85$ (1H, s, $\mathrm{H}-5), 11.79$ and $12.20\left(2 \mathrm{H}\right.$, each s, 2NH exchangeable with $\left.\mathrm{D}_{2} \mathrm{O}\right)$. Anal. Calcd. for $\mathrm{C}_{17} \mathrm{H}_{11} \mathrm{~N}_{3} \mathrm{O}_{4}$ (321.29): C, 63.55; H, 3.45; N, 13.08. Found C, 63.28; H, 3.95; N, 13.10.

\section{References}

1. Ungwitayatorn, J.; Samee, W.; Pimthon, J. J. Mol. Struct. 2004, 689, 99.

2. Ishakava, T.; Oku, Y.; Tanaka, T.; Kumamoto, T. Tetrahedron Lett. 1999, 40, 3777.

3. Xu, Z. Q.; Buckheit, R. W.; Stup, T. L.; Flavin, M. T.; Khilevich, A.; Rizzo, J. D.; Lin, L.; Zembower, D. E. Bioorg. Med. Chem. Lett. 1998, 8, 2179.

4. Mazzei, M.; Sottofattori, E.; Dondero, R.; Ibrahim, M.; Melloni, E.; Michetti, M. Farmaco 1999, 53, 452.

5. Puccetti, L.; Fasolis, G.; Vullo, D.; Chohan, Z. H.; Scozzafava A.; Supuran, C. T. Bioorg. Med. Chem. Lett. 2005, 15, 3096.

6. Modranka, J. N.; Nawrot, E.; Graczyk, J. Eur. J. Med. Chem. 2006, 41, 1301.

7. Göker, H.; Boykin, D. W.; Y1ld1z, S. Bioorg. Med. Chem. 2005, 13, 1707.

8. Ali, T. E. Phosphorus, Sulfur Silicon Relat. Elem. 2007, 182, 1717. 
9. Al-Nakib, T.; Benjak, V.; Meegan, M. J.; Chandy, R. Eur. J. Med. Chem. 1990, $25,455$.

10. Albrecht, U.; Lalk, M.; Langer, P. Bioorg. Med. Chem. 2005, 13, 1531.

11. Zhao, P.-L.; Li, J.; Yang, G.-Fu. Bioorg. Med. Chem. 2007, 15, 1888.

12. Oganesyan, E. T.; Tuskayev, V. A.; Sarkisov, L. S. Khim-Farm. Zh. 1994, $28,17$.

13. Dauzonne, D.; Folleas, B.; Martinez, L.; Chabot, G. G. Eur. J. Med. Chem. 1997, 32, 71.

14. Wernar, L.; Norbert, M. J. Heterocycl. Chem. 1996, 33, 943.

15. Wilfried, De N.; Jeffery, E.; Carleen, E.; Nick, S.; Christine, B. N.; Thomas, C. Int. J. Radiat. Oncol. Biol. phys. 1990, 18, 1359, Chem. Abst. 1991, 114, 20251.

16. Singh, G.; Singh, R.; Girdhar, N. K.; Ishar, M. P. S. Tetrahedron 2002, 58, 2471.

17. Chang, C.; Wu, C.; Kuo, S.; Wang, J.; Teng, C. Chin. Pharm. J. 2002, 54, 127.

18. Ibrahim, S. S.; Sami, S. M.; Abdel-Halim, A. M.; Laly, Y. Indian J. Chem. 1986, $25 B, 384$.

19. Nohara, A.; Kuriki, H.; Saija, T.; Ukawn, K.; Murata, T.; Kanno, M.; Sanno, Y. J. Med. Chem. 1975, 18, 34.

20. Nohara, A.; Kuriki, H.; Sugihara, H.; Kanno, M.; Sanno, Y. J. Med. Chem. 1977, $20,141$.

21. Arnold, C.; Thatcher, D. N. J. Org. Chem. 1967, 45, 1014.

22. Peterson, U.; Heitzer, H. Liebigs Ann. Chem. 1976, 1659.

23. Ibrahim, M. A. Tetrahedron 2009, 65, 7687.

24. Ibrahim, M. A. Synth. Commun. 2009, 39, 3527. 